\title{
Nonpremixed Flameless Combustion in Furnace: Influence of Burner Configuration
}

\author{
Guochang Wang ${ }^{a}$, Kin-Pang Cheong ${ }^{b^{*}}$, Jicang Si ${ }^{a}$ and Jianchun $\mathrm{Mi}^{{ }^{*}}$ \\ ${ }^{a}$ College of Engineering, Peking University, Beijing 100871, China \\ ${ }^{\mathrm{b}}$ School of Aeronautics and Astronautics, Sichuan University, Chengdu, 610065, China \\ *Correspondence to: jmi@pku.edu.cn; kpcheong@scu.edu.cn
}

Section 1. Effect of $k-\varepsilon$ model and EDC model constants on CFD simulation Section 2. Present simulated temperature and $\mathrm{OH}$ concentration fields 


\section{Section 1. Effect of $k-\varepsilon$ model and EDC model constants on CFD simulation}

(a) OFO-1.1

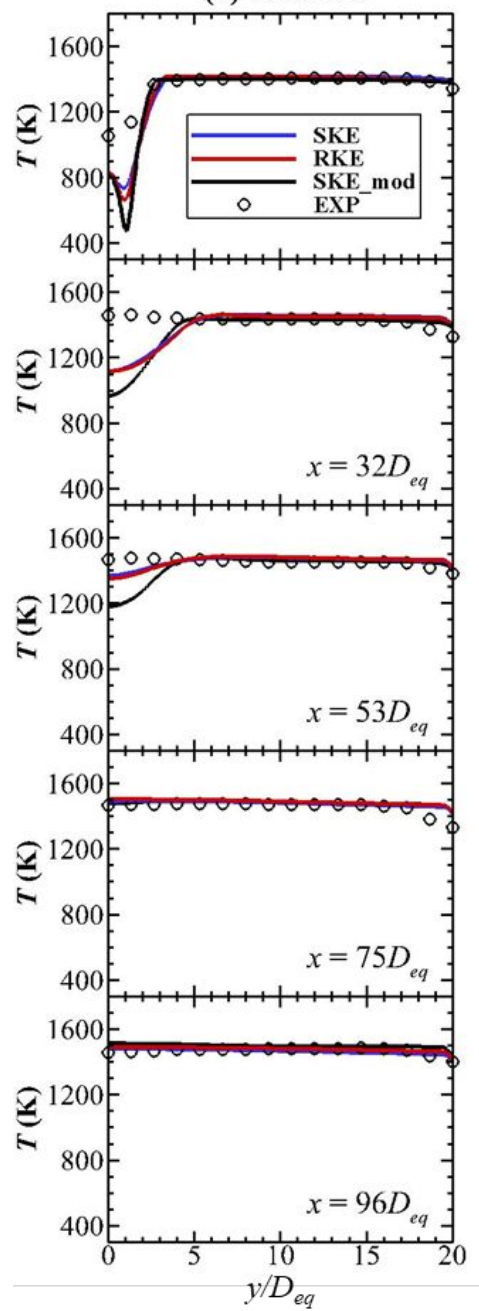

(b) OFO-6

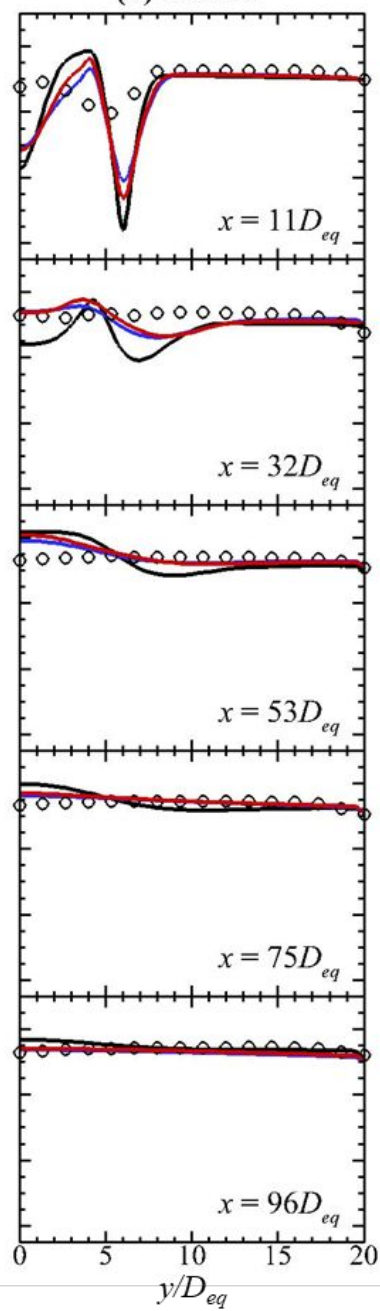

(c) FOF-1.1



(d) FOF-6

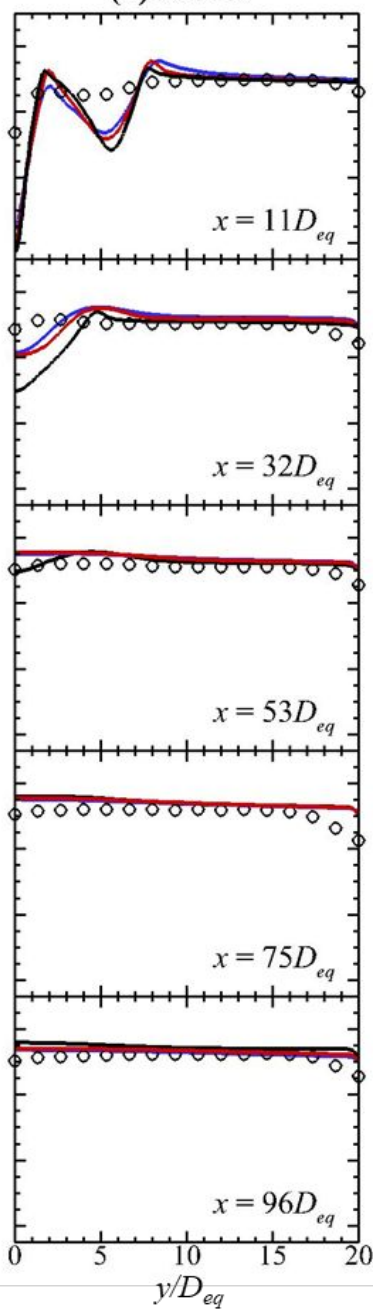

Figure S1. Effect of turbulent model on the CFD prediction of the temperature distributions for the OFO-1.1, OFO-6, FOF-1.1 and FOF-6 cases at $P_{i n}=15 \mathrm{~kW}$ and $\Phi=0.9$. SKE, RKE and SKE_mod are the results from standard, realizable and modified $k-\varepsilon$ model (where $C_{1 \varepsilon}=1.6$ ) and EXP represents the experimental data. The standard $k-\varepsilon$ model is chosen in the present study. 
(a) OFO-1.1

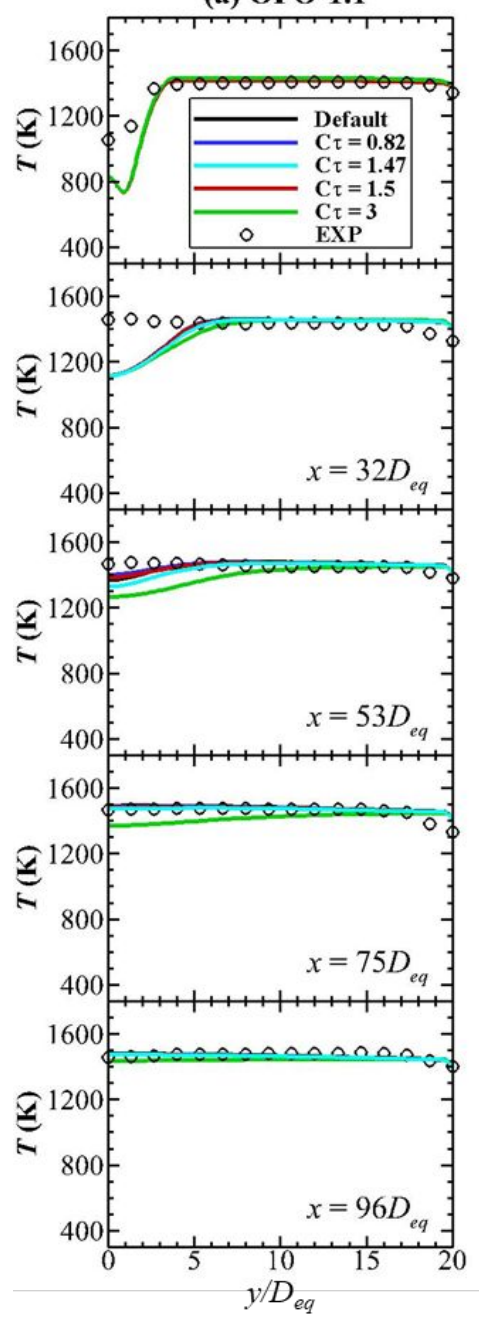

(b) OFO-6

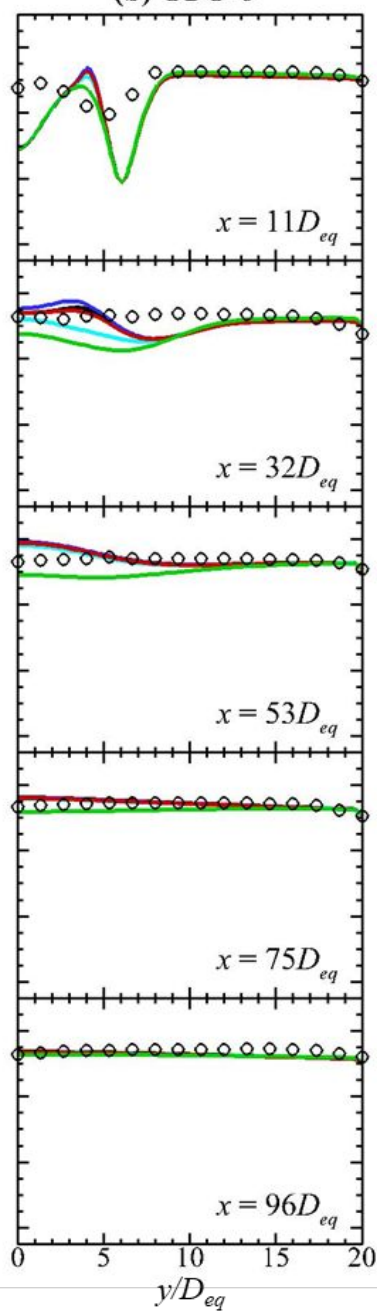

(c) FOF-1.1



(d) FOF-6

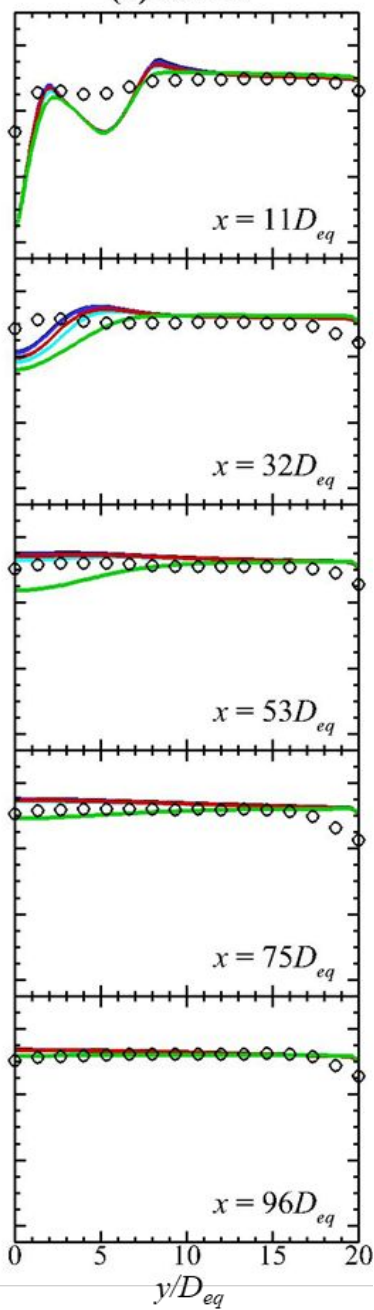

Figure S2. Effect of EDC model constants on the CFD prediction of the temperature distributions for the

OFO-1.1, OFO-6, FOF-1.1 and FOF- 6 cases at $P_{i n}=15 \mathrm{~kW}$ and $\Phi=0.9$. The default values $\left(C_{\tau}=0.4083, C_{\xi}\right.$

$=2.1377)$ and those from Aminian et $\mathrm{al}^{32}\left(C_{\tau}=1.5, C_{\xi}=2.14\right.$ ), Patente et al ${ }^{34}$ (two sets: $C_{\tau}=1.47, C_{\xi}=1.90$ and $\left.C_{\tau}=0.82, C_{\xi}=2.14\right)$ and Evans et $\mathrm{al}^{33}\left(C_{\tau}=3, C_{\xi}=1\right)$ are tested. The default values are chosen in the present study. 


\section{Section 2. Present simulated temperature and $\mathrm{OH}$ concentration fields}

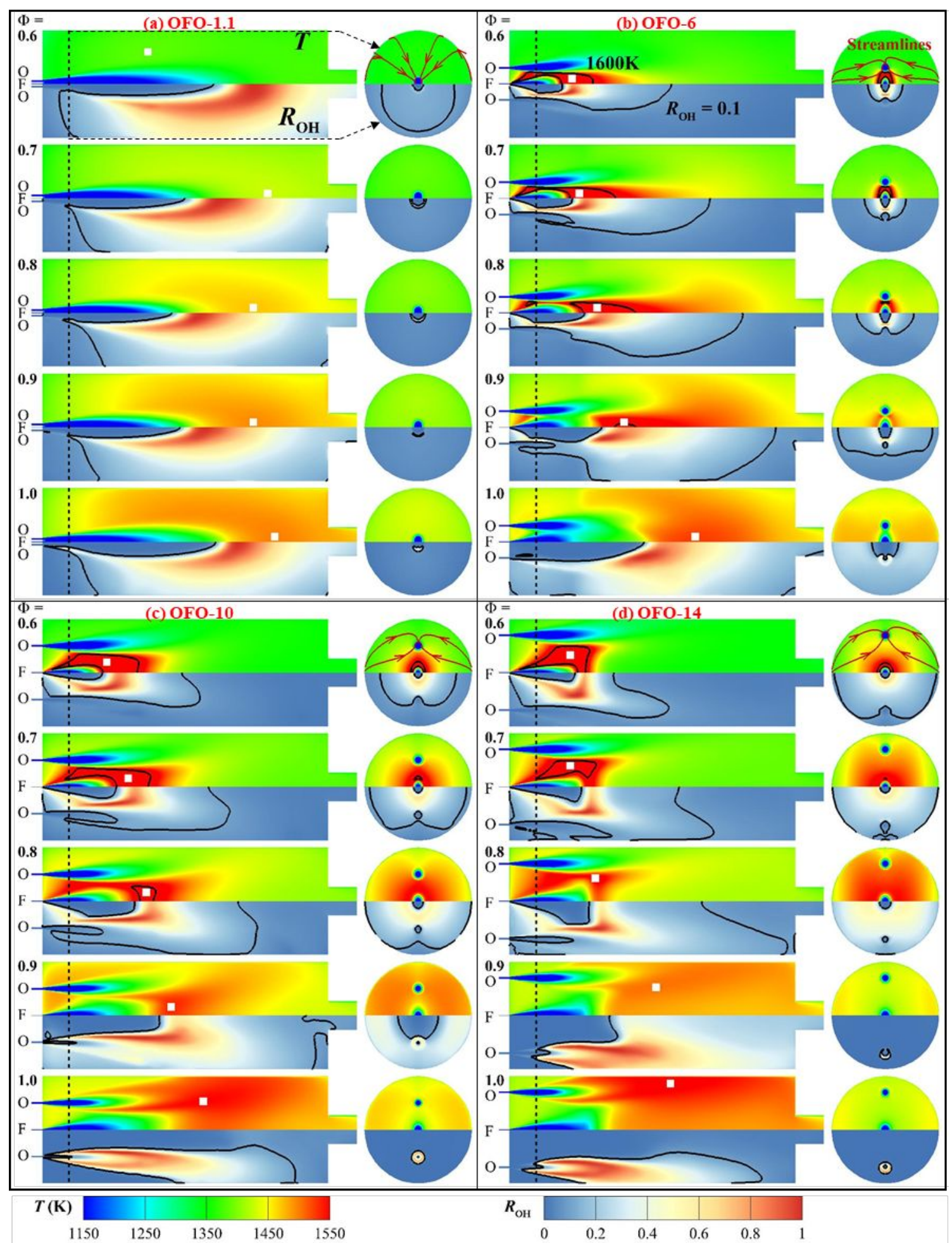

Figure S3. Longitudinal ( $x y$ ) center-plane and cross-sectional ( $y z$ ) distributions of $T$ (upper) and $R_{\mathrm{OH}}$ (lower) at $x=10 D_{e q}$ for the OFO-1.1, OFO-6, OFO-10, OFO-14 cases at $\boldsymbol{P}_{\boldsymbol{i n}}=\mathbf{1 5} \mathbf{k W}$ and $\Phi=0.6 \sim 1.0$. Black lines mark the boundaries of the HTR and RZ by $T=1600 \mathrm{~K}$ and $R_{\mathrm{OH}}=0.1$, respectively. White square dots 
show the position of $T_{\max }$ while some streamlines are shown at $\Phi=0.6$.



Figure S4. Longitudinal ( $x y$ ) center-plane and cross-sectional ( $y z$ ) distributions of $T$ (upper) and $R_{\mathrm{OH}}$ (lower) at $x=10 D_{e q}$ for the OFO-2.7 and OFO-4.3 cases at $\boldsymbol{P}_{\boldsymbol{i n}}=\mathbf{1 5} \mathbf{~ k W}$ and $\Phi=0.6 \sim 1.0$. Black lines mark the boundaries of the HTR and RZ by $T=1600 \mathrm{~K}$ and $R_{\mathrm{OH}}=0.1$, respectively. White square dots show the position of $T_{\max }$ while some streamlines are shown at $\Phi=0.6$. 




Figure S5. Longitudinal ( $x y$ ) center-plane and cross-sectional ( $y z$ ) distributions of $T$ (upper) and $R_{\mathrm{OH}}$ (lower) at $x=10 D_{e q}$ for the FOF-1.1, FOF-6, FOF-10, FOF-14 cases at $\boldsymbol{P}_{\boldsymbol{i n}}=\mathbf{1 5} \mathbf{k W}$ and $\Phi=0.6 \sim 1.0$. Black lines mark the boundaries of the HTR and RZ by $T=1600 \mathrm{~K}$ and $R_{\mathrm{OH}}=0.1$, respectively. White square dots show the position of $T_{\max }$ while some streamlines are shown at $\Phi=0.6$. 


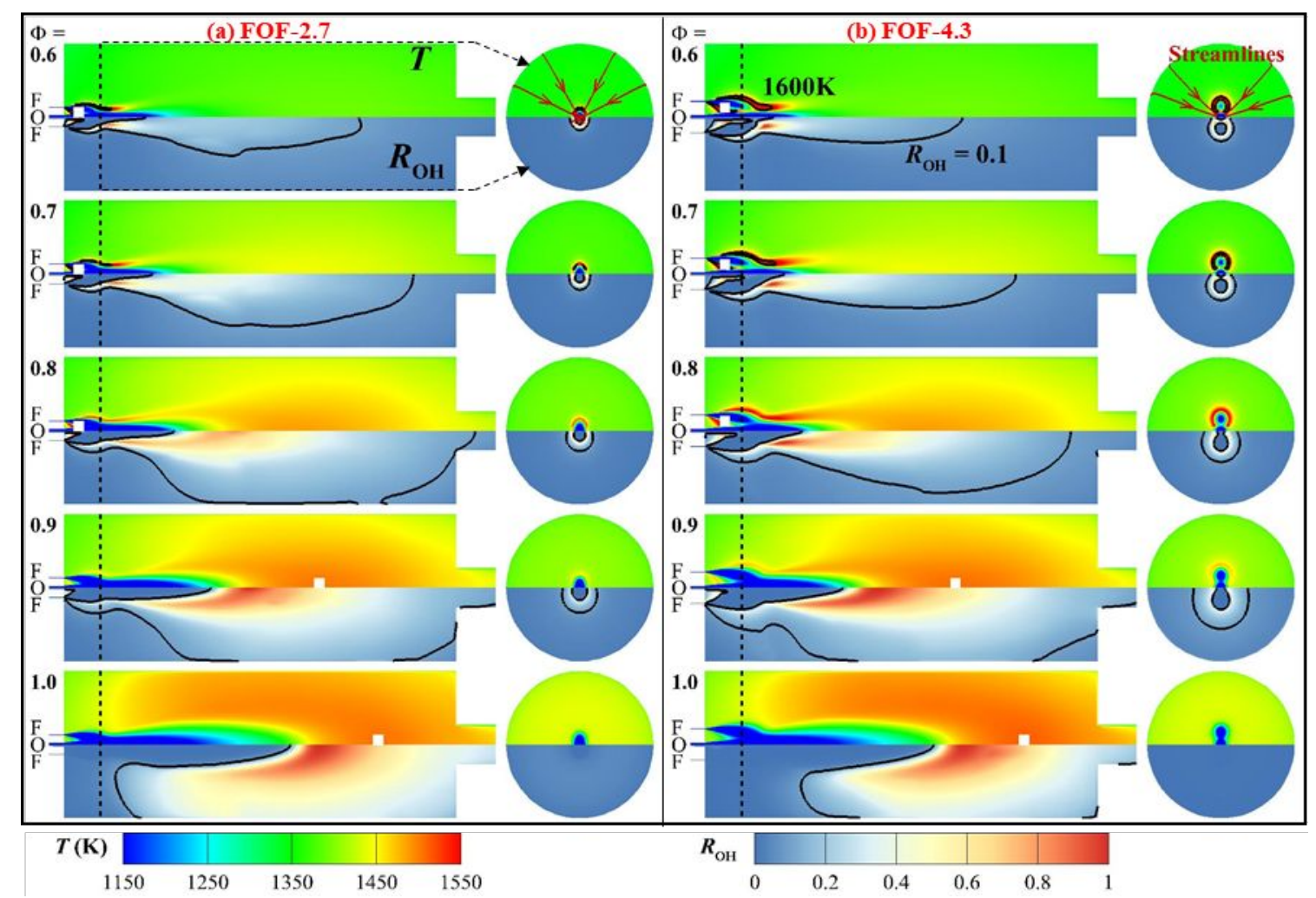

Figure S6. Longitudinal ( $x y$ ) center-plane and cross-sectional ( $y z$ ) distributions of $T$ (upper) and $R_{\mathrm{OH}}$ (lower) at $x=10 D_{e q}$ for the FOF-2.7 and FOF-4.3 cases at $\boldsymbol{P}_{\boldsymbol{i n}}=\mathbf{1 5} \mathbf{~ k W}$ and $\Phi=0.6 \sim 1.0$. Black lines mark the boundaries of the HTR and RZ by $T=1600 \mathrm{~K}$ and $R_{\mathrm{OH}}=0.1$, respectively. White square dots show the position of $T_{\max }$ while some streamlines are shown at $\Phi=0.6$. 




Figure S7. Longitudinal ( $x y$ ) center-plane and cross-sectional ( $y z$ ) distributions of $T$ (upper) and $R_{\mathrm{OH}}$ (lower) at $x=10 D_{e q}$ for the OFO-1.1, OFO-6, OFO-10, OFO-14 cases at $\boldsymbol{P}_{\boldsymbol{i n}}=\mathbf{1 0} \mathbf{k W}$ and $\Phi=0.6 \sim 1.0$. Black lines mark the boundaries of the HTR and RZ by $T=1600 \mathrm{~K}$ and $R_{\mathrm{OH}}=0.1$, respectively. White square dots show the position of $T_{\max }$ while some streamlines are shown at $\Phi=0.6$. 




Figure S8. Longitudinal ( $x y$ ) center-plane and cross-sectional ( $y z$ ) distributions of $T$ (upper) and $R_{\mathrm{OH}}$ (lower) at $x=10 D_{e q}$ for the FOF-1.1, FOF-6, FOF-10, FOF-14 cases at $\boldsymbol{P}_{\boldsymbol{i n}}=\mathbf{1 0} \mathbf{k W}$ and $\Phi=0.6 \sim 1.0$. Black lines mark the boundaries of the HTR and RZ by $T=1600 \mathrm{~K}$ and $R_{\mathrm{OH}}=0.1$, respectively. White square dots show the position of $T_{\max }$ while some streamlines are shown at $\Phi=0.6$. 